\title{
ON SMOOTH SOLUTIONS OF THE CAUCHY PROBLEM IN ONE-DIMENSIONAL NONLINEAR THERMOELASTICITY*
}

\author{
BY \\ WILLIAM J. HRUSA AND MICHAEL A. TARABEK \\ Carnegie Mellon University
}

1. Introduction. In the absence of dissipation, smooth motions of nonlinear elastic materials will generally develop singularities in finite time (cf. [6, 7]). For nonlinear thermoelastic materials, Slemrod [9] established global existence and decay of classical solutions to certain initial-boundary value problems, with smooth and small data, in one spatial dimension. Slemrod's proof, which is based on Matsumura's refinement [8] of the classical energy method, makes crucial use of Poincaré's inequality and consequently does not apply to situations in which the interval occupied by the body is unbounded. Subsequently, Zheng and Shen $[10,11]$ studied global existence of smooth solutions to the Cauchy problem in which a thermoelastic body occupies the entire real line. In [10] they use Fourier analysis to obtain precise decay rates for spatial $L^{p}$-norms (with $p=1,2, \infty$ ) of solutions to the linearized equations. Then, in [11], they combine weighted energy estimates with the results of [10] to establish global existence in the nonlinear case; they also obtain precise decay rates for the solution. (See also [12].) Because they use both Fourier analysis and energy estimates they impose assumptions of $L^{1}$-type and of $L^{2}$-type on the initial data (e.g., the initial velocity is assumed to be small in $W^{1,1}(\mathbf{R})$ and in $H^{3}(\mathbf{R})$ ).

In this paper we show how the energy method alone can be used to establish global existence and decay of classical solutions to the Cauchy problem with smooth and small data. We combine certain estimates of Slemrod that remain valid on unbounded spatial intervals with some additional ones that compensate for the loss of Poincaré's inequality. The additional estimates exploit some relations associated with the second law of thermodynamics. Our theorem is stated in Sec. 3 and the proof is given in Sec. 4. Some background material on one-dimensional thermoelasticity is presented in Sec. 2.

The equations of thermoelasticity have special structure that is induced by the second law of thermodynamics. By exploiting this structure we eliminate the need to obtain decay estimates for the linearized problem. This not only leads to a substantial reduction of work but also makes it unnecessary to assume that the initial data (or any of their derivatives) belong to $L^{1}(\mathbf{R})$.

Zheng and Shen $[10,11]$ also discuss the system of radiation hydrodynamics. Our existence theorem can be applied to this system by making a simple change of

${ }^{*}$ Received September 7, 1988.

(C)1989 Brown University 
variables. On the other hand, the approach of $[10,11]$ can be applied to certain other hyperbolic-parabolic systems having less structure than the one considered here.

We note that a globally defined classical solution should not be expected if the data are large. Indeed, the work of Coleman and Gurtin [1] shows that in a onedimensional nonlinear thermoelastic body acceleration waves of small initial amplitude decay, but waves of large initial amplitude can explode in finite time; in other words, the damping effect of thermal diffusion manages to restrain waves of small amplitude but the destabilizing effect of a nonlinear elastic response is dominant for waves of large amplitude. Moreover, for specialized constitutive equations, Dafermos and Hsiao [4] have shown that if the initial data are large then the solution to the Cauchy problem will develop singularities in finite time.

2. One-dimensional thermoelasticity. We consider a homogeneous one-dimensional body that occupies the interval $B$ in a (fixed) reference configuration and has unit reference density. For such a body, the laws of balance of momentum, balance of energy, and growth of entropy take the forms

$$
\begin{gathered}
u_{t t}=\sigma_{x}+f, \\
e_{t}=-q_{x}+\sigma \varepsilon_{t}+r, \\
\eta_{t} \geq \frac{r}{\theta}-\left(\frac{q}{\theta}\right)_{x},
\end{gathered}
$$

with

$$
\begin{aligned}
& u \text { the displacement, } \\
& \varepsilon:=u_{x} \text { the strain, } \\
& \sigma \text { the stress, } \\
& f \text { the body force, } \\
& e \text { the internal energy, } \\
& q \text { the heat flux, } \\
& r \text { the heat supply, } \\
& \eta \text { the entropy, and } \\
& \theta \text { the absolute temperature. }
\end{aligned}
$$

Each of the above fields is assumed to be a smooth function of the material point $x \in B$ and the time $t$; the strain and temperature are required to satisfy

$$
\varepsilon>-1, \quad \theta>0 .
$$

Subscripts $x$ and $t$ indicate partial derivatives.

We define the free energy $\psi$ through

$$
\psi:=e-\theta \eta
$$

and we write

$$
g:=\theta_{x}
$$


for the temperature gradient. Then (2.2) and (2.3) may be combined to give the free-energy inequality

$$
\psi_{t}+\eta \theta_{t}-\sigma \varepsilon_{t}+\frac{q g}{\theta} \leq 0
$$

A thermoelastic material is described by constitutive relations that express $\psi, \sigma$, $\eta$, and $q$ as functions of $(\varepsilon, \theta, g)$; we assume that these constitutive functions are smooth and that the heat flux is given by Fourier's law

$$
q(x, t)=-\kappa(\varepsilon(x, t), \theta(x, t)) g(x, t) .
$$

The second law of thermodynamics places restrictions on the constitutive relations for $\psi, \sigma$, and $\eta$, and requires that the thermal conductivity $\kappa$ be positive. Specifically, it follows from theorems of Coleman and Noll [2] and Coleman and Mizel [3] that the free energy is independent of the temperature gradient:

$$
\psi(x, t)=\hat{\psi}(\varepsilon(x, t), \theta(x, t))
$$

that the stress and entropy are determined by $\hat{\psi}$ through the relations

$$
\begin{gathered}
\sigma(x, t)=\hat{\psi}_{\varepsilon}(\varepsilon(x, t), \theta(x, t)), \\
\eta(x, t)=-\hat{\psi}_{\theta}(\varepsilon(x, t), \theta(x, t)) ;
\end{gathered}
$$

and that

$$
\kappa \geq 0 .
$$

It follows from (2.5), (2.9), and (2.11) that the internal energy is given by

$$
e=\hat{\psi}(\varepsilon, \theta)-\theta \hat{\psi}_{\theta}(\varepsilon, \theta) \text {. }
$$

An important consequence of (2.9), (2.10), (2.11) is the identity

$$
\psi_{t}+\eta \theta_{t}-\sigma \varepsilon_{t}=0
$$

which is equivalent to Gibb's relation

$$
e_{t}-\theta \eta_{t}-\sigma \varepsilon_{t}=0
$$

by virtue of (2.5). In view of (2.15) the equation of balance of energy (2.2) can be rewritten as

$$
\theta \eta_{t}=-q_{x}+r
$$

The restrictions (2.9) through (2.12) ensure the free-energy inequality (2.7) is satisfied and further that $(2.3)$ is satisfied whenever (2.2) holds. Indeed, it follows immediately from $(2.14),(2.8)$, and (2.12) that

$$
\psi_{t}+\eta \theta_{t}-\sigma \varepsilon_{t}+\frac{q g}{\theta}=-\frac{\kappa(\varepsilon, \theta) g^{2}}{\theta} \leq 0 .
$$

Moreover, if (2.2) holds then (2.7) is equivalent to (2.3).

The elastic modulus, the stress-temperature modulus, and the specific heat play important roles in thermoelasticity; these moduli can be expressed in terms of $\hat{\psi}$ as follows:

$$
\hat{\psi}_{\varepsilon \varepsilon}(\varepsilon, \theta) \text { is the elastic modulus, }
$$

$\hat{\psi}_{\varepsilon \theta}(\varepsilon, \theta)$ is the stress-temperature modulus, 
and $-\theta \hat{\psi}_{\theta \theta}(\varepsilon, \theta)$ is the specific heat.

We refer to the recent book of Day [5] for a much more complete discussion of one-dimensional thermoelasticity.

3. The Cauchy problem. We assume now that the body occupies the entire real line in its reference configuration (i.e. $B=\mathbf{R}$ ) and that the heat flux, free energy, stress, entropy, and internal energy are given by $(2.8)-(2.11),(2.13)$. We seek a smooth solution to the balance laws $(2.1),(2.2)$ when the initial values of the displacement, velocity, and temperature are prescribed. For simplicity, we assume that the body force $f$ and the heat supply $r$ both vanish identically. Thus we consider the initialvalue problem

$$
\begin{gathered}
u_{t t}=\hat{\psi}_{\varepsilon \varepsilon}\left(u_{x}, \theta\right) u_{x x}+\hat{\psi}_{\varepsilon \theta}\left(u_{x}, \theta\right) \theta_{x}, \\
-\theta \hat{\psi}_{\theta \theta}\left(u_{x}, \theta\right) \theta_{t}=\left(\kappa\left(u_{x}, \theta\right) \theta_{x}\right)_{x}+\theta \hat{\psi}_{\varepsilon \theta}\left(u_{x}, \theta\right) u_{x t}, \quad x \in \mathbf{R}, t \geq 0, \\
u(x, 0)=u_{0}(x), \quad u_{t}(x, 0)=u_{1}(x), \quad \theta(x, 0)=\theta_{0}(x), \quad x \in \mathbf{R} .
\end{gathered}
$$

where $u_{0}, u_{1}$, and $\theta_{0}$ are given functions.

We assume there is a temperature $\theta^{*}$ such that when $\varepsilon=0$ and $\theta=\theta^{*}$ the elastic modulus, specific heat, and thermal conductivity are strictly positive, and the stresstemperature modulus is nonzero, i.e.

$$
\begin{array}{cl}
\hat{\psi}_{\varepsilon \varepsilon}\left(0, \theta^{*}\right)>0, & -\theta^{*} \hat{\psi}_{\theta \theta}\left(0, \theta^{*}\right)>0, \quad \kappa\left(0, \theta^{*}\right)>0 \\
& \hat{\psi}_{\varepsilon \theta}\left(0, \theta^{*}\right) \neq 0 .
\end{array}
$$

If the initial data are smooth and $u_{0}^{\prime}, u_{1}, \theta_{0}-\theta^{*}$ are small (in appropriate norms) then (3.1), (3.2), (3.3) has a unique globally defined classical solution. More precisely we have the following result. (In the theorem below, derivatives should be interpreted in the distributional sense and $H^{k}(\mathbf{R})$ denotes the usual Sobolev space consisting of those functions in $L^{2}(\mathbf{R})$ whose derivatives through order $k$ belong to $L^{2}(\mathbf{R})$.)

Theorem. Let $\theta^{*} \in(0, \infty)$ be given. Assume that $\hat{\psi} \in C^{4}((-1, \infty) \times(0, \infty)), k \in$ $C^{3}((-1, \infty) \times(0, \infty))$ and that $(3.4),(3.5)$ hold. Then there is a number $\mu>0$ such that whenever $u_{0}, u_{1}, \theta_{0}: \mathbf{R} \rightarrow \mathbf{R}$ satisfy

$$
u_{0}^{\prime} \in H^{2}(\mathbf{R}), \quad u_{1} \in H^{3}(\mathbf{R}), \quad \theta_{0}-\theta^{*} \in H^{4}(\mathbf{R})
$$

and $^{1}$

$$
\left\|u_{0}^{\prime}\right\|_{H^{2}}^{2}+\left\|u_{1}\right\|_{H^{3}}^{2}+\left\|\theta_{0}-\theta^{*}\right\|_{H^{4}}^{2} \leq \mu^{2}
$$

then the initial-value problem (3.1), (3.2), (3.3) has a unique solution $(u, \theta)$, with

$$
\begin{gathered}
u_{x}, u_{t}, u_{x x}, u_{x t}, u_{t t}, u_{x x x x}, u_{x x t}, u_{x t t}, u_{t t t}, \\
\theta-\theta^{*}, \theta_{x}, \theta_{t}, \theta_{x x}, \theta_{x t}, \theta_{t l}, \theta_{x x x}, \theta_{x x t} \in C\left([0, \infty) ; L^{2}(\mathbf{R})\right) \cap L^{\infty}\left([0, \infty) ; L^{2}(\mathbf{R})\right), \\
u_{x x}, u_{x t}, u_{t t}, u_{x x x x}, u_{x x t}, u_{x t t}, u_{t t t}, \\
\theta_{x x}, \theta_{t}, \theta_{x x x}, \theta_{x t}, \theta_{t l}, \theta_{x x x x}, \theta_{x x t}, \theta_{x t t} \in L^{2}\left([0, \infty) ; L^{2}(\mathbf{R})\right)
\end{gathered}
$$

\footnotetext{
${ }^{1}$ The Sobolev embedding theorem and (3.7) with $\mu$ sufficiently small imply $u_{0}^{\prime}>-1$ and $\theta_{0}>0$.
} 
and

$$
u_{x}(x, t)>-1, \quad \theta(x, t)>0, \quad x \in \mathbf{R}, \quad t \geq 0 .
$$

REMARKS.

1. It follows from (3.8), (3.9) and standard embedding theorems that

$$
\begin{gathered}
u \in C^{2}(\mathbf{R} \times[0, \infty)), \quad \theta \in C^{1}(\mathbf{R} \times[0, \infty)), \\
\theta_{x x} \in C(\mathbf{R} \times[0, \infty)),
\end{gathered}
$$

and that, as $t \rightarrow \infty$,

$$
\begin{gathered}
u_{x}, u_{t}, u_{x x}, u_{x t}, u_{t t}, \theta-\theta^{*}, \theta_{x}, \theta_{t}, \theta_{x x}, \theta_{x t} \rightarrow 0 \quad \text { uniformly on } \mathbf{R}, \\
u_{x x}, u_{x t}, u_{t t}, \theta_{x}, \theta_{t}, \theta_{x x}, \theta_{x t} \rightarrow 0 \quad \text { in } L^{2}(\mathbf{R}) .
\end{gathered}
$$

2. A solution satisfying (3.8), (3.9), (3.10) can be obtained under slightly weakened hypotheses on the data. Moreover, solutions with different regularity can be obtained under different assumptions of smoothness on $\hat{\psi}, \kappa$, and the data (e.g., for $\hat{\psi} \in$ $C^{5}, \kappa \in C^{4}$ a global smooth solution exists if $u_{0}^{\prime}, u_{1}, \theta-\theta^{*} \in H^{3}(\mathbf{R})$ with small norms). These issues will not be pursued further. Our theorem has been formulated so that we may employ as many of Slemrod's estimates as possible in the proof.

3. An analogous theorem holds if Fourier's law (2.8) is replaced by the more general constitutive equation

$$
q=\hat{q}(\varepsilon, \theta, g)
$$

with $\hat{q}(\cdot, \cdot, 0)=0$ and $\hat{q}_{g}\left(0, \theta^{*}, 0\right)<0$. Our proof would require only minor changes.

4. The theorem can be modified to accommodate a nonzero body force $f$ and a nonzero heat supply $r$ such that

$$
\begin{gathered}
f, r \in L^{1}\left([0, \infty) ; L^{2}(\mathbf{R})\right), \\
f_{x}, f_{t}, f_{x x}, f_{x t}, f_{t t}, r_{x}, r_{t}, r_{x x}, r_{x t}, r_{t t} \in L^{2}\left([0, \infty) ; L^{2}(\mathbf{R})\right)
\end{gathered}
$$

with sufficiently small norms.

5. Zheng and Shen [11] assume that (3.4), (3.5) hold and that

$$
u_{0}^{\prime}, u_{1}, \theta-\theta^{*} \in H^{3}(\mathbf{R}) \cap W^{1,1}(\mathbf{R})
$$

with small norms in both spaces. They obtain global existence and give precise decay rates for various norms of the solution.

6. Slemrod [9] established global existence for the cases when $B=[0,1]$ and the boundary conditions are either

$$
\sigma(0, t)=\sigma(1, t)=0, \quad \theta(0, t)=\theta(1, t)=\theta^{*}
$$

or

$$
u(0, t)=u(1, t)=q(0, t)=q(1, t)=0 .
$$

For simplicity he assumes the heat flux is given by

$$
q=\hat{q}(g)
$$


where $\hat{q}(0)=0$ and $\hat{q}^{\prime}(0)<0$; with only minor changes his proof applies when $q$ is given by (3.14). It is interesting to note that Slemrod's argument does not apply to the boundary conditions

$$
u(0, t)=u(1, t)=0, \quad \theta(0, t)=\theta(1, t)=\theta^{*}
$$

because they lead to ill-behaved boundary terms in a crucial energy integral.

4. Proof of global existence. Throughout this section we assume that $\theta^{*} \in(0, \infty)$ is fixed, that $\hat{\psi} \in C^{4}((-1, \infty) \times(0, \infty)), \kappa \in C^{3}((-1, \infty) \times(0, \infty))$, and that (3.4), (3.5), (3.6) hold. Moreover, without loss of generality we assume

$$
\hat{\psi}\left(0, \theta^{*}\right)=0 .
$$

Our proof of global existence is based on a priori estimates that can be used to continue a local solution globally in time. The existence of a local solution can be established by a standard contraction-mapping argument and we omit the details. The relevant result is recorded in the proposition below. In view of the local nature of assumptions (3.4), (3.5) we must ensure that $\left(u_{x}, \theta\right)$ remains in a suitable neighborhood of $\left(0, \theta^{*}\right)$. We therefore choose $\delta \in(0, \infty)$ such that

$$
\delta<\min \left(1, \frac{\theta^{*}}{2}\right)
$$

and $\hat{\psi}_{\varepsilon \varepsilon},\left|\hat{\psi}_{\varepsilon \theta}\right|,-\hat{\psi}_{\theta \theta}, \kappa$ are bounded away from zero on

$$
\mathscr{O}:=(-\delta, \delta) \times\left(\theta^{*}-\delta, \theta^{*}+\delta\right),
$$

i.e.

$$
\begin{aligned}
& m_{1}:=\inf _{\digamma} \hat{\psi}_{\varepsilon \varepsilon}>0, \quad m_{2}:=\inf _{\digamma}\left|\hat{\psi}_{\varepsilon \theta}\right|>0 \\
& m_{3}:=\inf _{c}\left(-\hat{\psi}_{\theta \theta}\right)>0, \quad m_{4}:=\inf _{\digamma} \kappa>0 .
\end{aligned}
$$

Proposition. Let $u_{0}, u_{1}, \theta_{0}: \mathbf{R} \rightarrow \mathbf{R}$ be given with

$$
u_{0}^{\prime} \in H^{2}(\mathbf{R}), \quad u_{1} \in H^{3}(\mathbf{R}), \quad \theta_{0}-\theta^{*} \in H^{4}(\mathbf{R})
$$

and

$$
\left(u_{0}^{\prime}(x), \theta_{0}(x)\right) \in \mathscr{\odot} \quad \forall x \in \mathbf{R} .
$$

Then the initial-value problem $(3.1),(3.2),(3.3)$ has a unique solution $(u, \theta)$ on a maximal time interval $[0, T), 0<T \leq \infty$, with

$$
\begin{gathered}
u_{x}, u_{t}, u_{x x}, u_{x t}, u_{t t}, u_{x x x x}, u_{x x t}, u_{x t t}, u_{t t t}, \theta-\theta^{*}, \\
\theta_{x}, \theta_{t}, \theta_{x x}, \theta_{x t}, \theta_{t t}, \theta_{x x x}, \theta_{x x t} \in C\left([0, T) ; L^{2}(\mathbf{R})\right), \\
\theta_{x t t} \in L_{\mathrm{loc}}^{2}\left([0, T) ; L^{2}(\mathbf{R})\right), \\
\left(u_{x}(x, t), \theta(x, t)\right) \in \mathscr{G} \quad \forall x \in \mathbf{R}, t \in[0, T) .
\end{gathered}
$$


Moreover if

$$
\begin{aligned}
\sup _{t \in[0, T)} \int_{-\infty}^{\infty}\left\{u_{x}^{2}+\right. & u_{t}^{2}+u_{x x}^{2}+u_{x t}^{2}+u_{t t}^{2}+u_{x x x}^{2}+u_{x x t}^{2} \\
+ & u_{x t t}^{2}+u_{t t t}^{2}+\left(\theta-\theta^{*}\right)^{2}+\theta_{x}^{2}+\theta_{t}^{2} \\
+\theta_{x x}^{2}+\theta_{x t}^{2} & \left.+\theta_{t t}^{2}+\theta_{x x x}^{2}+\theta_{x x t}^{2}\right\}(x, t) d x \\
& +\int_{0}^{T} \int_{-\infty}^{\infty} \theta_{x t t}^{2}(x, s) d x d s<\infty
\end{aligned}
$$

and

$$
\sup _{\mathbf{R} \times[0, T)}\left|u_{x}\right|<\delta, \quad \sup _{\mathbf{R} \times[0, T)}\left|\theta-\theta^{*}\right|<\delta
$$

then $T=\infty$.

We now consider the local solution $(u, \theta)$ given by the proposition. Our goal is to show that if (3.7) is satisfied with $\mu$ sufficiently small then (4.10), (4.11) hold. For this purpose, we set

$$
\begin{gathered}
U_{0}:=\left\|u_{0}^{\prime}\right\|_{H^{2}}^{2}+\left\|u_{1}\right\|_{H^{3}}^{2}+\left\|\theta-\theta^{*}\right\|_{H^{4}}^{2}, \\
\mathscr{E}(t):=\max _{s \in[0, t]} \int_{-\infty}^{\infty}\left\{u_{x}^{2}+u_{t}^{2}+u_{x x}^{2}+u_{x t}^{2}+u_{t t}^{2}+u_{x x x}^{2}+u_{x x t}^{2}\right. \\
+u_{x t t}^{2}+u_{t t t}^{2}+\left(\theta-\theta^{*}\right)^{2}+\theta_{x}^{2}+\theta_{t}^{2} \\
\left.+\theta_{x x}^{2}+\theta_{x t}^{2}+\theta_{t t}^{2}+\theta_{x x x}^{2}+\theta_{x x t}^{2}\right\}(x, s) d x \\
+\int_{0}^{t} \int_{-\infty}^{\infty}\left\{u_{x x}^{2}+u_{x t}^{2}+u_{t t}^{2}+u_{x x x}^{2}+u_{x x t}^{2}+u_{x t t}^{2}\right. \\
+u_{t t t}^{2}+\theta_{x}^{2}+\theta_{t}^{2}+\theta_{x x}^{2}+\theta_{x t}^{2} \quad \forall t \in[0, T) .
\end{gathered}
$$

We shall show that if $U_{0}$ is sufficiently small then

$$
\mathscr{E}(t) \leq \frac{1}{4} \delta^{2} \quad \forall t \in[0, T)
$$

Since, by the Sobolev embedding theorem,

$$
\left\{u_{x}^{2}+\left(\theta-\theta^{*}\right)^{2}\right\}(x, t) \leq \mathscr{E}(t) \quad \forall x \in \mathbf{R}, t \in[0, T),
$$

the inequality (4.14) implies that (4.10), (4.11) hold and hence that $T=\infty$.

The desired bound for $\mathscr{E}$ will be obtained from a chain of energy estimates. To express these estimates in a concise form, it is convenient to define

$$
\nu(t):=\sup _{\substack{x \in \mathbf{R} \\ s \in[0, t]}}\left\{u_{x x}^{2}+u_{x t}^{2}+\theta_{x}^{2}+\theta_{t}^{2}\right\}^{1 / 2}(x, s) \quad \forall t \in[0, T),
$$


and to introduce

$$
\begin{aligned}
& A(x, t):=\hat{\psi}_{\varepsilon \varepsilon}\left(u_{x}(x, t), \theta(x, t)\right) \\
& B(x, t):=\hat{\psi}_{\varepsilon \theta}\left(u_{x}(x, t), \theta(x, t)\right) \\
& C(x, t):=-\theta(x, t) \hat{\psi}_{\theta \theta}\left(u_{x}(x, t), \theta(x, t)\right) \\
& D(x, t):=\kappa\left(u_{x}(x, t), \theta(x, t)\right) \\
& E(x, t):=\theta(x, t) \hat{\psi}_{\varepsilon \theta}\left(u_{x}(x, t), \theta(x, t)\right) \quad \forall x \in \mathbf{R}, t \geq 0,
\end{aligned}
$$

so that the system (3.1), (3.2) can be rewritten as

$$
\begin{gathered}
u_{t t}=A u_{x x}+B \theta_{x}, \\
C \theta_{t}=\left(D \theta_{x}\right)_{x}+E u_{x t},
\end{gathered}
$$

with

$$
\begin{gathered}
A(x, t) \geq m_{1}, \quad|B(x, t)| \geq m_{2}, \\
C(x, t) \geq \frac{\theta^{*}}{2} m_{3}, \quad D(x, t) \geq m_{4} \\
|E(x, t)| \geq \frac{\theta^{*}}{2} m_{2} \quad \forall x \in \mathbf{R}, t \in[0, T) .
\end{gathered}
$$

For future use, we note that differentiation of (4.18), (4.19) with respect to $x$ yields

$$
\begin{gathered}
u_{x t t}=A u_{x x x}+B \theta_{x x}+A_{x} u_{x x}+B_{x} \theta_{x} \\
C \theta_{x t}=D \theta_{x x x}+E u_{x x t}-C_{x} \theta_{t}+2 D_{x} \theta_{x x}+D_{x x} \theta_{x}+E_{x} u_{x t}
\end{gathered}
$$

while differentiation with respect to $t$ yields

$$
\begin{gathered}
u_{t t t}=A u_{x x t}+B \theta_{x t}+A_{t} u_{x x}+B_{t} \theta_{x} \\
C \theta_{t t}=D \theta_{x x t}+E u_{x t t}-C_{t} \theta_{t}+D_{t} \theta_{x x}+D_{x} \theta_{x t}+D_{x t} \theta_{x}+E_{t} u_{x t}
\end{gathered}
$$

In the calculations that follow, we make repeated use of the elementary inequalities

$$
\begin{gathered}
|\alpha \beta| \leq \frac{1}{2}\left(\alpha^{2}+\beta^{2}\right), \\
\left(\sum_{i=1}^{N} \alpha_{i}\right)^{2} \leq N \sum_{i=1}^{N} \alpha_{i}^{2},
\end{gathered}
$$

and we use the symbol $\Gamma$ to denote a (possibly large) generic positive constant that is independent of $u_{0}, u_{1}, \theta_{0}$, and $T$. (We note that $\Gamma$ is allowed to depend on $\theta^{*}, \delta, m_{1}, m_{2}, m_{3}, m_{4}$, and on bounds for $\kappa, \hat{\psi}$, and their derivatives on the compact set $\overline{\mathscr{O}} \subset(-1, \infty) \times(0, \infty)$.) The inequality

$$
\frac{\theta^{*}}{2}<\theta(x, t)<\frac{3 \theta^{*}}{2} \quad \forall x \in \mathbf{R}, t \in[0, T),
$$

which follows from (4.2), (4.3), and (4.9), will also be used. The arguments involved in our derivation of the estimate $(4.43)$ below are very similar to ones employed by Slemrod [9]; many of the details will therefore be omitted. 
To obtain our first energy integral we multiply (4.21) by $\theta u_{x t},(4.22)$ by $\theta_{x}$, add the resulting equations and integrate over $\mathbf{R} \times[0, t]$. After performing several integrations by parts, and recalling that $E=\theta B$, we arrive at the identity

$$
\begin{gathered}
\frac{1}{2} \int_{-\infty}^{\infty}\left\{A \theta u_{x x}^{2}+\theta u_{x t}^{2}+C \theta_{x}^{2}\right\}(x, t) d x+\int_{0}^{t} \int_{-\infty}^{\infty} D \theta_{x x}^{2}(x, s) d x d s \\
=\frac{1}{2} \int_{-\infty}^{\infty}\left\{A \theta u_{x x}^{2}+\theta u_{x t}^{2}+C \theta_{x}^{2}\right\}(x, 0) d x \\
+\int_{0}^{t} \int_{-\infty}^{\infty}\left\{\frac{1}{2} \theta A_{t} u_{x x}^{2}+\frac{1}{2} \theta_{t} A u_{x x}^{2}+\frac{1}{2} \theta_{t} u_{x t}^{2}+\frac{1}{2} C_{t} \theta_{x}^{2}\right. \\
\quad-A \theta_{x} u_{x x} u_{x t}-B \theta_{x}^{2} u_{x t}-C_{x} \theta_{x} \theta_{t} \\
\left.-D_{x} \theta_{x} \theta_{x x}+E_{x} \theta_{x} u_{x t}\right\}(x, s) d x d s .
\end{gathered}
$$

It follows that (4.20) and (4.27) that the left-hand side of (4.28) is bounded from below by

$$
\frac{\theta^{*}}{4} \int_{-\infty}^{\infty}\left\{m_{1} u_{x x}^{2}+u_{x t}^{2}+m_{3} \theta_{x}^{2}\right\}(x, t) d x+m_{4} \int_{0}^{t} \int_{-\infty}^{\infty} \theta_{x x}^{2}(x, s) d x d s
$$

for all $t \in[0, T)$. After some routine estimations on the right-hand side we conclude that (4.28) yields the inequality

$$
\begin{gathered}
\int_{-\infty}^{\infty}\left\{u_{x x}^{2}+u_{x t}^{2}+\theta_{x}^{2}\right\}(x, t) d x+\int_{0}^{t} \int_{-\infty}^{\infty} \theta_{x x}^{2}(x, s) d x d s \\
\leq \Gamma U_{0}+\Gamma \nu(t) E(t) \quad \forall t \in[0, T) .
\end{gathered}
$$

To give an indication of the steps used to estimate the right-hand side of (4.28) we give the details for two typical terms. Let $L$ be an upper bound for $\left|\hat{\psi}_{\varepsilon \varepsilon}\right|+\left|\kappa_{\varepsilon}\right|+\left|\kappa_{\theta}\right|$ on $\mathscr{O}$. Employing (3.3), (4.6), (4.17), and (4.27) we find that

$$
\begin{aligned}
\int_{-\infty}^{\infty} A \theta u_{x x}^{2}(x, 0) d x & =\int_{-\infty}^{\infty} \hat{\psi}_{\varepsilon \varepsilon}\left(u_{0}^{\prime}(x), \theta_{0}(x)\right) \theta_{0}(x) u_{0}^{\prime \prime}(x)^{2} d x \\
& \leq \frac{3}{2} \theta^{*} L \int_{-\infty}^{\infty} u_{0}^{\prime \prime}(x)^{2} d x \leq \Gamma U_{0}
\end{aligned}
$$

and using (4.9), (4.17), and (4.25), we see that

$$
\begin{aligned}
& -\int_{0}^{t} \int_{-\infty}^{\infty} D_{x} \theta_{x} \theta_{x x}(x, s) d x d s \\
& \quad \leq \int_{0}^{t} \int_{-\infty}^{\infty}\left|\theta_{x}\left\{\kappa_{\varepsilon}\left(u_{x}, \theta\right) u_{x x} \theta_{x x}+\kappa_{\theta}\left(u_{x}, \theta\right) \theta_{x} \theta_{x x}\right\}(x, s)\right| d x d s \\
& \quad \leq L \nu(t) \int_{0}^{t} \int_{-\infty}^{\infty}\left\{\left|u_{x x} \theta_{x x}\right|+\left|\theta_{x} \theta_{x x}\right|\right\}(x, s) d x d s \\
& \quad \leq \frac{L}{2} \nu(t) \int_{0}^{t} \int_{-\infty}^{\infty}\left\{u_{x x}^{2}+\theta_{x}^{2}+2 \theta_{x x x}^{2}\right\}(x, s) d x d s \\
& \quad \leq \Gamma \nu(t) E(t) \quad \forall t \in[0, T) .
\end{aligned}
$$

The other terms on the right-hand side of (4.28) can be estimated in a similar fashion. 
We now multiply $(4.23)$ by $\theta u_{t t},(4.24)$ by $\theta_{t}$, and proceed as in the derivation of (4.29) to infer that

$$
\begin{gathered}
\int_{-\infty}^{\infty}\left\{u_{x t}^{2}+u_{t t}^{2}+\theta_{t}^{2}\right\}(x, t) d x+\int_{0}^{t} \int_{-\infty}^{\infty} \theta_{x t}^{2}(x, s) d x d s \\
\leq \Gamma U_{0}+\Gamma \nu(t) E(t) \quad \forall t \in[0, T) .
\end{gathered}
$$

Here we have used Eqs. (4.18), (4.19) to express the initial values of $u_{t t}$ and $\theta_{t}$ in terms of $u_{0}^{\prime}, u_{0}^{\prime \prime}, u_{1}^{\prime}, \theta_{0}, \theta_{0}^{\prime}$, and $\theta_{0}^{\prime \prime}$.

Energy integrals of higher order can be obtained ${ }^{2}$ by differentiating Eqs. (4.21), (4.22) with respect to $x,(4.23),(4.24)$ with respect to $t,(4.21),(4.22)$ with respect to $t$, and using appropriate multipliers. These energy integrals yield the inequality

$$
\begin{aligned}
\int_{-\infty}^{\infty}\{ & \left.u_{x x x}^{2}+u_{x x t}^{2}+u_{x t t}^{2}+u_{t t t}^{2}+\theta_{x x}^{2}+\theta_{x t}^{2}+\theta_{t t}^{2}\right\}(x, t) d x \\
& +\int_{0}^{t} \int_{-\infty}^{\infty}\left\{\theta_{x x x}^{2}+\theta_{x x t}^{2}+\theta_{x t t}^{2}\right\}(x, s) d x d s \\
\leq & \Gamma U_{0}+\Gamma\left\{\nu(t)+\nu(t)^{4}\right\} \mathscr{E}(t) \quad \forall t \in[0, T) .
\end{aligned}
$$

The derivation of (4.33) is very similar to Slemrod's derivation ${ }^{3}$ of (3.12) of [10] and his subsequent estimation of the right-hand side.

The next group of estimates will be obtained by using Eqs. (4.21) through (4.24) to express various derivatives of $u$ and $\theta$ in terms of quantities that have already been estimated.

Applying (4.26) to (4.22) we find that

$$
D^{2} \theta_{x x x}^{2} \leq 6\left\{C^{2} \theta_{x t}^{2}+E^{2} u_{x x t}^{2}+C_{x}^{2} \theta_{t}^{2}+4 D_{x}^{2} \theta_{x x}^{2}+D_{x x}^{2} \theta_{x}^{2}+E_{x}^{2} u_{x t}^{2}\right\} .
$$

We integrate (4.34) over $\mathbf{R}$ and use (4.33) to conclude that

$$
\int_{-\infty}^{\infty} \theta_{x x x}^{2}(x, t) d x \leq \Gamma U_{0}+\Gamma\left\{\nu(t)+\nu(t)^{4}\right\} \mathscr{E}(t) \quad \forall t \in[0, T) .
$$

By the same kind of argument, (4.24) and (4.33) yield

$$
\int_{-\infty}^{\infty} \theta_{x x t}^{2}(x, t) d x \leq \Gamma U_{0}+\Gamma\left\{\nu(t)+\nu(t)^{4}\right\} \mathscr{E}(t) \quad \forall t \in[0, T),
$$

while (4.22), (4.32), and (4.33) yield

$$
\int_{0}^{t} \int_{-\infty}^{\infty} u_{x x t}^{2}(x, s) d x d s \leq \Gamma U_{0}+\Gamma\left\{\nu(t)+\nu(t)^{4}\right\} \mathscr{E}(t) \quad \forall t \in[0, T),
$$

and (4.23), (4.32), and (4.37) give

$$
\int_{0}^{t} \int_{-\infty}^{\infty} u_{t t t}^{2}(x, s) d x d s \leq \Gamma U_{0}+\Gamma\left\{\nu(t)+\nu(t)^{4}\right\} \mathscr{E}(t) .
$$

${ }^{2}$ This procedure is formal because the regularity of the local solution is insufficient to validate several of the steps. However, rigorous derivations of the desired energy integrals can be achieved easily by using difference quotients and taking limits.

${ }^{3}$ Slemrod does not use the energy integral that arises from differentiating (4.21), (4.22) with respect to $x$. Use of this energy integral could also be avoided here, but that would lead to extra work later on. 
A bound for $\int_{0}^{t} \int_{-\infty}^{\infty} u_{x t t}^{2}$ can now be obtained by interpolation. Indeed the identity ${ }^{4}$

$$
\begin{aligned}
\int_{0}^{t} \int_{-\infty}^{\infty} u_{x t t}^{2}(x, s) d x d s= & \int_{-\infty}^{\infty} u_{t t} u_{x x t}(x, 0) d x-\int_{-\infty}^{\infty} u_{t t} u_{x x t}(x, t) d x \\
& +\int_{0}^{t} \int_{-\infty}^{\infty} u_{x x t} u_{t t t}(x, s) d x d s
\end{aligned}
$$

implies the inequality

$$
\begin{aligned}
\int_{0}^{t} \int_{-\infty}^{\infty} u_{x t t}^{2}(x, s) d x d s \leq & \frac{1}{2} \int_{-\infty}^{\infty}\left\{u_{t t}^{2}+u_{x x t}^{2}\right\}(x, 0) d x \\
& +\frac{1}{2} \int_{-\infty}^{\infty}\left\{u_{t t}^{2}+u_{x x t}^{2}\right\}(x, t) d x \\
& +\frac{1}{2} \int_{0}^{t} \int_{-\infty}^{\infty}\left\{u_{x x t}^{2}+u_{t t t}^{2}\right\}(x, s) d x d s \quad \forall t \in[0, T)
\end{aligned}
$$

which, in conjunction with (4.32), (4.33), (4.37), and (4.38), yields

$$
\int_{0}^{t} \int_{-\infty}^{\infty} u_{x t t}^{2}(x, s) d x d s \leq \Gamma U_{0}+\Gamma\left\{\nu(t)+\nu(t)^{4}\right\} \mathscr{E}(t) \quad \forall t \in[0, T) .
$$

Using Eqs. (4.21) and (4.24), together with (4.26), (4.29), (4.33), and (4.41), we find that

$$
\int_{0}^{t} \int_{-\infty}^{\infty}\left\{u_{x x x}^{2}+\theta_{t t}^{2}\right\}(x, s) d x d s \leq \Gamma U_{0}+\Gamma\left\{\nu(t)+\nu(t)^{4}\right\} \mathscr{E}(t) \quad \forall t \in[0, T) .
$$

By combining (4.29), (4.32), (4.33), (4.35), (4.36), (4.37), (4.38), (4.41), and (4.42) we conclude that

$$
\begin{aligned}
& \int_{-\infty}^{\infty}\left\{u_{x x}^{2}+u_{x t}^{2}+u_{t t}^{2}+u_{x x x}^{2}+u_{x x t}^{2}+u_{x t t}^{2}+u_{t t t}^{2}\right. \\
& \left.+\theta_{x}^{2}+\theta_{t}^{2}+\theta_{x x}^{2}+\theta_{x t}^{2}+\theta_{t t}^{2}+\theta_{x x x}^{2}+\theta_{x x t}^{2}\right\}(x, s) d x \\
& +\int_{0}^{t} \int_{-\infty}^{\infty}\left\{u_{x x x}^{2}+u_{x x t}^{2}+u_{x t t}^{2}+u_{t t t}^{2}\right. \\
& \left.+\theta_{x x}^{2}+\theta_{x t}^{2}+\theta_{t t}^{2}+\theta_{x x x}^{2}+\theta_{x x t}^{2}+\theta_{x t t}^{2}\right\}(x, s) d x d s \\
& \leq \Gamma U_{0}+\Gamma\left\{\nu(t)+\nu(t)^{4}\right\} \mathscr{E}(t) \quad \forall t \in[0, T) .
\end{aligned}
$$

A similar bound for

$$
\int_{-\infty}^{\infty}\left\{u_{x}^{2}+u_{t}^{2}+\left(\theta-\theta^{*}\right)^{2}\right\}(x, t) d x+\int_{0}^{t} \int_{-\infty}^{\infty}\left\{u_{x x}^{2}+u_{x t}^{2}+u_{t t}^{2}+\theta_{x}^{2}+\theta_{t}^{2}\right\}(x, s) d x d s
$$

will be obtained by exploiting some identities associated with the free-energy inequality.

It follows that $(2.16)$ with $r \equiv 0$ that

$$
\left(\theta-\theta^{*}\right) \eta_{t}=-\left(\theta-\theta^{*}\right) \frac{q_{x}}{\theta} .
$$

\footnotetext{
${ }^{4}$ This identity can be verified formally via integration by parts. It can be derived rigorously using difference
} quotients or mollifiers. 
Using (2.8), (2.14), (4.44), and (2.1) with $f \equiv 0$ we conclude that

$$
\frac{\partial}{\partial t}\left(\psi+\left(\theta-\theta^{*}\right) \eta-\sigma^{*} \varepsilon+\frac{1}{2} u_{t}^{2}\right)+\frac{\theta^{*}}{\theta^{2}} \kappa\left(u_{x}, \theta\right) \theta_{x}^{2}=\frac{\partial}{\partial x}\left[\left(\sigma-\sigma^{*}\right) u_{t}-\left(\theta-\theta^{*}\right) \frac{q}{\theta}\right],
$$

where $\sigma^{*}:=\hat{\psi}_{\varepsilon}\left(0, \theta^{*}\right)$ is the residual stress. Integration of $(4.45)$ over $\mathbf{R} \times[0, t]$ produces

$$
\begin{gathered}
\int_{-\infty}^{\infty}\left\{\Lambda\left(u_{x}, \theta\right)+\frac{1}{2} u_{t}^{2}\right\}(x, t) d x+\theta^{*} \int_{0}^{t} \int_{-\infty}^{\infty} \frac{\kappa\left(u_{x}, \theta\right)}{\theta^{2}} \theta_{x}^{2}(x, s) d x d s \\
=\int_{-\infty}^{\infty}\left\{\Lambda\left(u_{0}^{\prime}, \theta_{0}\right)+\frac{1}{2} u_{1}^{2}\right\}(x) d x \quad \forall t \in[0, T),
\end{gathered}
$$

where we have put

$$
\Lambda(\varepsilon, \theta):=\hat{\psi}(\varepsilon, \theta)-\hat{\psi}_{\varepsilon}\left(0, \theta^{*}\right) \varepsilon-\left(\theta-\theta^{*}\right) \hat{\psi}_{\theta}(\varepsilon, \theta) .
$$

It follows from (3.4) and (4.1) that

$$
\begin{gathered}
\Lambda\left(0, \theta^{*}\right)=\Lambda_{\varepsilon}\left(0, \theta^{*}\right)=\Lambda_{\theta}\left(0, \theta^{*}\right)=0, \\
\Lambda_{\varepsilon \varepsilon}\left(0, \theta^{*}\right)>0, \quad \Lambda_{\varepsilon \theta}\left(0, \theta^{*}\right)=0, \quad \Lambda_{\theta \theta}\left(0, \theta^{*}\right)>0,
\end{gathered}
$$

and consequently there is a neighborhood $\mathscr{U}$ of $\left(0, \theta^{*}\right)$ in $\mathbf{R}^{2}$ such that

$$
\xi^{2}+\left(\lambda-\theta^{*}\right)^{2} \leq \Gamma \Lambda(\xi, \lambda)
$$

for all $(\xi, \lambda) \in \mathscr{U}$. An inequality of the form (4.49) actually holds for all $(\xi, \lambda) \in \mathscr{O}$. Indeed, using Taylor's theorem and (4.4) we find that

$$
\begin{gathered}
\Lambda(\xi, \lambda)-\hat{\psi}\left(\xi, \theta^{*}\right)+\hat{\psi}_{\varepsilon}\left(0, \theta^{*}\right) \xi \geq \frac{1}{2} m_{3}\left(\lambda-\theta^{*}\right)^{2} \quad \forall(\xi, \lambda) \in \mathscr{O}, \\
\hat{\psi}\left(\xi, \theta^{*}\right)-\hat{\psi}_{\varepsilon}\left(0, \theta^{*}\right) \xi \geq \frac{1}{2} m_{1} \xi^{2} \quad \forall(\xi, \lambda) \in \mathscr{O} .
\end{gathered}
$$

Adding the inequalities (4.50) and (4.51), and recalling (4.9), we arrive at

$$
\Lambda\left(u_{x}(x, t), \theta(x, t)\right) \geq \frac{1}{2}\left\{m_{1} u_{x}^{2}+m_{3}\left(\theta-\theta^{*}\right)^{2}\right\}(x, t) \quad \forall x \in \mathbf{R}, t \in[0, T) .
$$

The identity (4.46) therefore yields the a priori bound

$$
\int_{-\infty}^{\infty}\left\{u_{x}^{2}+u_{t}^{2}+\left(\theta-\theta^{*}\right)^{2}\right\}(x, t) d x+\int_{0}^{t} \int_{-\infty}^{\infty} \theta_{x}^{2}(x, s) d x d s \leq \Gamma U_{0} \quad \forall t \in[0, T) .
$$

We now multiply (4.18) by $u_{t t} / A$ and integrate the result over $\mathbf{R} \times[0, t]$, using integration by parts, to obtain

$$
\begin{aligned}
\int_{0}^{t} \int_{-\infty}^{\infty} A^{-1} u_{t t}^{2}(x, s) d x d s-\int_{0}^{t} \int_{-\infty}^{\infty} u_{x t}^{2}(x, s) d x d s \\
\quad=\int_{-\infty}^{\infty} u_{0}^{\prime} u_{1}^{\prime}(x) d x-\int_{-\infty}^{\infty} u_{x} u_{x t}(x, t) d x \\
\quad+\int_{0}^{t} \int_{-\infty}^{\infty} A^{-1} B u_{t t} \theta_{x}(x, s) d x d s \quad \forall t \in[0, T) .
\end{aligned}
$$


Similarly, we multiply (4.19) by $u_{x t} / C$ and integrate the result to obtain

$$
\begin{gathered}
\int_{0}^{t} \int_{-\infty}^{\infty} C^{-1}|E| u_{x t}^{2}(x, s) d x d s=\gamma \int_{-\infty}^{\infty} u_{1} \theta_{0}^{\prime}(x) d x-\gamma \int_{-\infty}^{\infty} u_{t} \theta_{x}(x, t) d x \\
+\gamma \int_{0}^{t} \int_{-\infty}^{\infty}\left\{u_{t t} \theta_{x}-C^{-1} D u_{x t} \theta_{x x}\right. \\
\left.-C^{-1} D_{x} u_{x t} \theta\right\}(x, s) d x d s \\
\forall t \in[0, T),
\end{gathered}
$$

where $\gamma$ is either one or minus one, depending on the sign of $\hat{\psi}_{\varepsilon \theta}$ on $\mathscr{\sigma}$. The estimate

$$
\begin{aligned}
& \int_{0}^{t} \int_{-\infty}^{\infty}\left\{u_{x t}^{2}+u_{t t}^{2}\right\}(x, s) d x d s \leq \Gamma U_{0}+\Gamma\left\{\nu(t)+\nu(t)^{4}\right\} \mathscr{E}(t) \\
&+\Gamma \int_{0}^{t} \int_{-\infty}^{\infty}\left\{\left|u_{x t} \theta_{x x}\right|+\left|u_{t t} \theta_{x}\right|\right\}(x, s) d x d s \\
& \forall t \in[0, T]
\end{aligned}
$$

follows from adding a suitable multiple of (4.55) to (4.54) and using (4.43), (4.53). By applying the inequality

$$
|\alpha \beta| \leq \lambda \alpha^{2}+\frac{1}{4 \lambda} \beta^{2}, \quad \lambda>0
$$

with $\lambda$ sufficiently small and using (4.43), (4.53), we see that (4.56) yields

$$
\int_{0}^{t} \int_{-\infty}^{\infty}\left\{u_{x t}^{2}+u_{t t}^{2}\right\}(x, s) d x d s \leq \Gamma U_{0}+\Gamma\left\{\nu(t)+\nu(t)^{4}\right\} \mathscr{E}(t) \quad \forall t \in[0, T) .
$$

Applying (4.26) to (4.18) and (4.19) and using (4.43), (4.53), (4.58) we conclude that

$$
\int_{0}^{t} \int_{-\infty}^{\infty}\left\{u_{x x}^{2}+\theta_{t}^{2}\right\}(x, s) d x d s \leq \Gamma U_{0}+\Gamma\left\{\nu(t)+\nu(t)^{4}\right\} \mathscr{E}(t) \quad \forall t \in[0, T) .
$$

We are now ready to complete the proof. By combining (4.43), (4.53), (4.58) and (4.59) we arrive at

$$
\mathscr{E}(t) \leq \bar{\Gamma} U_{0}+\bar{\Gamma}\left\{\nu(t)+\nu(t)^{4}\right\} \mathscr{E}(t) \quad \forall t \in[0, T)
$$

where $\bar{\Gamma}$ is a constant that is independent of $T$ and the data. We choose $\varepsilon \in(0, \infty)$ such that

$$
\varepsilon \leq \frac{1}{2} \delta^{2}, \quad \bar{\Gamma}\left\{\varepsilon^{1 / 2}+\varepsilon^{2}\right\} \leq \frac{1}{4}
$$

and we then choose $\mu \in(0, \infty)$ such that

$$
\bar{\Gamma} \mu^{2} \leq \frac{1}{4} \varepsilon
$$

and

$$
U_{0} \leq \mu^{2} \Rightarrow \mathscr{E}(0) \leq \frac{1}{2} \varepsilon
$$

Here $\delta$ is the number introduced in (4.2), (4.3), (4.4). Suppose now that (3.7) holds with the above choice of $\mu$. Then, we have $\bar{\Gamma} U_{0} \leq \frac{1}{4} \varepsilon$. Moreover, it follows from the Sobolev embedding theorem that

$$
\nu(t)^{2} \leq \mathscr{E}(t) \quad \forall t \in[0, T) .
$$


We therefore conclude from (4.60) and (4.61) that for each $t \in[0, T)$ with $\mathscr{E}(t) \leq \varepsilon$ we actually have $\mathscr{E}(t) \leq \frac{1}{2} \varepsilon$. Consequently, by continuity, we have

$$
\mathscr{E}(t) \leq \frac{1}{2} \varepsilon \leq \frac{1}{4} \delta^{2} \quad \forall t \in[0, T),
$$

since $\mathscr{E}(0) \leq \frac{1}{2} \varepsilon$. In view of $(4.15)$ and the definition of $\mathscr{E}$, the a priori bound (4.65) implies that (4.10) and (4.11) hold, and consequently $T=\infty$. Finally, it follows from the definitions of $\mathscr{E}$ and $\mathscr{O}$ that the solution satisfies (3.8), (3.9), and (3.10).

Acknowledgments. We are grateful to M. E. Gurtin for valuable discussions. This work was supported by the Air Force Office of Scientific Research under grant AFOSR-85-0307.

\section{REFERENCES}

[1] B. D. Coleman and M. E. Gurtin, Waves in materials with memory, III. Thermodynamic influences on the growth and decay of acceleration waves, Arch. Rational Mech. Anal. 19, 266-298 (1965)

[2] B. D. Coleman and W. Noll, The thermodynamics of elastic materials with heat condition and viscosity: Arch. Rational Mech. Anal. 13, 167-178 (1963)

[3] B. D. Coleman and V. J. Mizel, Thermodynamics and departure from Fourier's law of heat conduction, Arch. Rational Mech. Anal. 13, 245-261 (1963)

[4] C. M. Dafermos and L. Hsiao, Development of singularities in solutions of equations of nonlinear thermoelasticity, Quart. Appl. Math. 44, 463-474 (1986)

[5] W. A. Day, A commentary on Thermodynamics, Springer-Verlag, 1988

[6] P. D. Lax, Development of singularities of solutions of nonlinear hyperbolic differential equations, J. Math. Physics 5, 611-613 (1964)

[7] R. C. MacCamy and V. J. Mizel, Existence and non-existence in the large of solutions of quasilinear wave equations, Arch. Rational. Mech. Anal. 25, 299-320 (1967)

[8] A. Matsumura, Global existence and asymptotics of the solutions of the second-order quasilinear hyperbolic equations with first order dissipation, Publ. Res. Inst. Math. Sci., Kyoto University. Series A 13, 349-379 (1977)

[9] M. Slemrod, Global existence, uniqueness, and asymptotic stability of classical smooth solutions in one-dimensional noniinear thermoelasticity, Arch. Rational Mech. Anal. 76, 97-133 (1981)

[10] S. Zheng and W. Shen, $L^{p}$ decay' estimates of solutions to the Cauchy problem of hyperbolic-parabolic coupled systems, Scientia Sinica (to appear)

[11] S. Zheng and W. Shen, Global solutions to the Cauchy problem of a class of hyperbolic-parabolic coupled systems, Scientia Sinica (to appear)

[12] S. Zheng and W. Shen, Global solutions to the Cauchy problem of a class of hyperbolic-parabolic coupled systems, International Workshop on Applied Differential Equations (S. T. Xiao and F. Q. $\mathrm{Pu}$, eds.), World Scientific Publishing, 1986, pp. 335-338 